\title{
Effect of Community Opening on Road Traffic
}

\section{Shi Feng}

North China Electricity Power University, Baoding 071000, China;

\author{
1361214913 @qq.com
}

Keywords: variable cellular transport model, VISSIM simulation complex network model, road network traffic flow

\begin{abstract}
In this paper, we mainly study the influence of the community opening on the surrounding road traffic, and correspondingly, build a model for the vehicle traffic. Firstly, focusing on the overall road network structure of the community and its surrounding area before and after the community opening ,we establish the Variable Cell Transmission Model (VCTM model).In this model ,the roads will be segmented and a new concept--traffic flow is proposed based on the transfer characteristics of cellular .Furthermore, we characterize the road traffic capacity by means of three traffic flow state evaluation indexes which are the average travel speed, the traffic impedance and the congestion delay .

Secondly, with the help of MATLAB, we abstract the target community network structure into a complex network model, and then by inputting the three description parameters, namely the degree distribution, the average path length and the clustering coefficient into VCTM model and using the VISSIM software for simulation, we get the output of the three traffic flow state evaluation indexes, thus establishing the community vehicle traffic model.

Then, corresponding to the road network structures of different communities and their different surrounding environments, five complex network models are constructed, which are respectively named the closed community model, the regular network model, the random network model, the small world network and the scale-free network model. Among those models, the closed community model corresponds to the community opening anterior network structure and the other 4 characterize the road network structures after the opening of community. By inputting the 5 kinds of complex network traffic model into the community vehicle traffic model, we can quantitatively compare the effects of the opening of different types of residential area on the road traffic according to the results of the model. the influence of the opening and the opening of each type on the road traffic was quantitatively compared. Through analysis, we can get that the regular network model traffic carrying capacity is the worst, while the scale-free network traffic carrying capacity optimal.

Finally, through the comparison and analysis of the above results, we get the effects of the opening of different types of community on the road network structures and the road traffic capacity. Based on the results we have got, we put forward several rationalization proposals about the community opening to the urban planning and traffic management department.
\end{abstract}

\section{Background of the problem}

In February 21, 2016, the State Council issued 《A number of opinions on Further Strengthening the administration of city planning and construction》 ,in which the sixteenth one is about the promotion of block system , which means ,in principle, closed residential quarters will no longer be constructed and the completed residential area and the unit compounds should gradually open , has aroused widespread concern and discussion.

In addition to the opening of community may lead to security issues, one of the focuses of the discussion is: whether the community opening can achieve the optimization of network structure, improve the road capacity and improve the traffic conditions or not, and how to characterize the improvement effect. One view is that the closed area has destroyed the city road network structure and blocked the "capillary" of the city, which is easy to cause the city traffic jams. 
After the opening of the community, the density of the road network will be improved and the road area will be increased as a result of which the road traffic capacity will naturally improve.

While some other people think that traffic capacity is related to many other factors, such as the residential area, location, external and internal road conditions and so on, which can not be generalized.

Meanwhile, some one else believe that although the number of passable road increases as a result of community opening, accordingly, the number of vehicles in the area surrounding the intersection of the main road will also increase, which may also affect the traffic speed of the main road.

\section{Solution and application of the model}

After consulting a large number of blueprints of the residential road network structures and geographic locations, we obtain several representative design patterns as shown below:
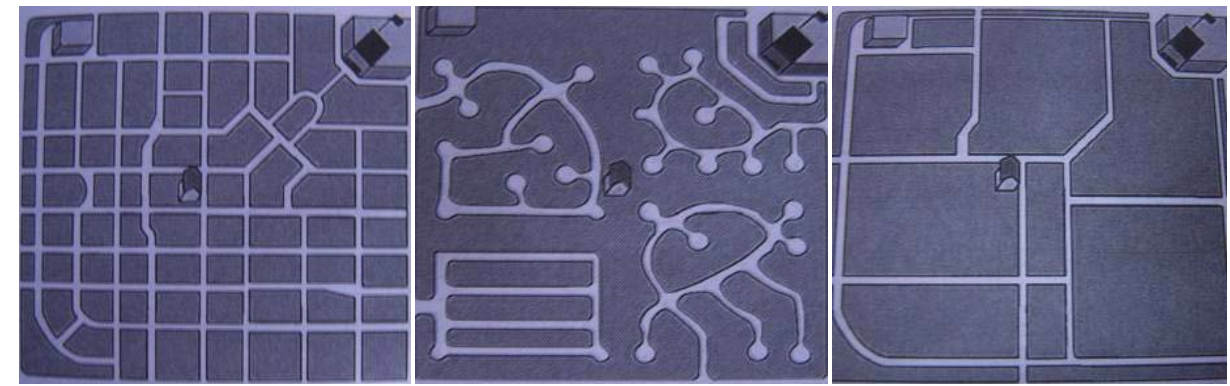

a. Network road structure b. arborescence road structure c. Highway road structure

Figure 1 Actual community road network structure diagram

Based on the actual community road network structure design shown above, we abstract the different types of the community and its surrounding road network structures into analog network ones, which cover all kinds of residential network structures relatively roundly, including five types mentioned above.

The designs are shown as below in Figure 2.

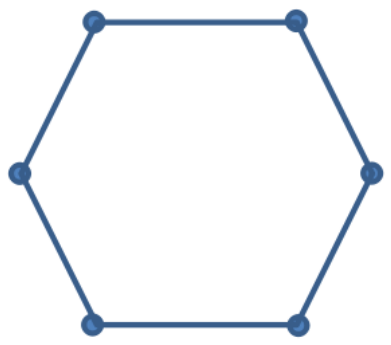

a. Closed network

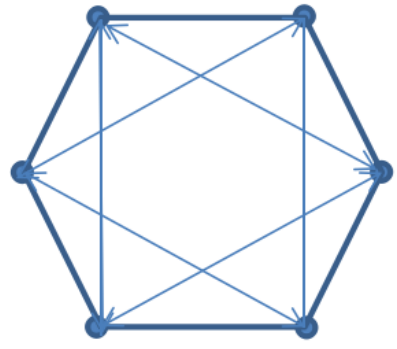

b. Regular network

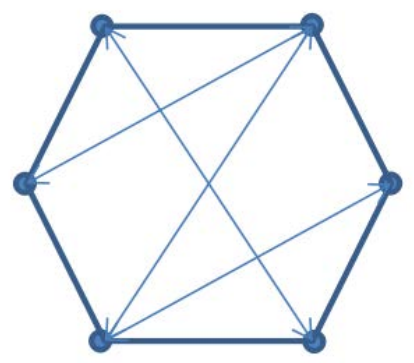

c. Random network

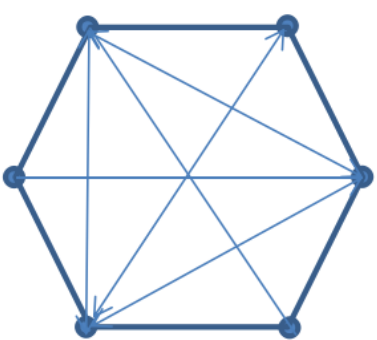

d. Small world network

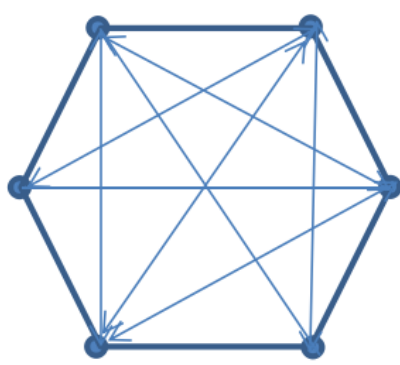

e. Scale-free network

Figure2 Abstracted design for community and its surrounding road network structure 


\section{Conclusion}

In this paper, we establish a Variable Cell Transmission Model and Complex Network model to analyze the influence of the opening of closed community on traffic capacity. Through the CTM model , we simulate five different types of network structures: closed community network, regular network, random network, small world network and scale-free network, among which the last four are for open communities.

By adjusting the structure parameters such as the probability of random rewiring probability, applying to Cellular Transport Model and combing with VISSIM traffic simulation software, we simulate the condition of the traffic flow and obtain the change situations of average speed, traffic impedance, hold plugging delay and other traffic flow parameters in different network traffic demand in order to analyze the traffic conditions of closed communities with different kinds of road network structures after their opening.

The results show us: Among the four kinds of network structure for opening communities, the effect of the scale-free network on the improvement of traffic conditions is the most obvious, which is followed by the random network and small world network, but for the regular network, the effect of the residential open on traffic condition improvement is the least obvious.

\section{References}

[1]Ling Zhao . Analysis of urban road network structure and its influence on traffic flow [D] Hunan: Central South University.2013

[2]Xiaojian Hu , Wei Wang , HuiSheng . Estimation of city traffic flow based onvariable cell transmission model [D]. Nanjing: Southeast University, 2010

[3]Wei Liu ,Qingshan Zeng, Braess paradox phenomenon based on complex network [J]. Henan: Zhengzhou University, 2015

[4] W. Strunk Jr., E.B. White, The Elements of Style, third ed., Macmillan, New York, 1979. Reference to a chapter in an edited book:

[5] G.R. Mettam, How to prepare an electronic version of your article, in: B.S. Jones, R.Z. Smith (Eds.), Introduction to the Electronic Age, E-Publishing Inc., New York, 1999, pp. 281-304.

[6] R.J. Ong, J.T. Dawley and P.G. Clem: submitted to Journal of Materials Research (2003)

[7] P.G. Clem, M. Rodriguez, J.A. Voigt and C.S. Ashley, U.S. Patent 6,231,666. (2001)

[8] Information on http://www.weld.labs.gov.cn 